\title{
BIBLIOGRAFIA
}

\section{Fichas para la Bibliografía de Poe en Hispanoamérica}

\author{
A D V E R T E N C I A
}

$\mathbf{E}^{N}$

N 1934 John Eugene Englekirk publicó, por medio del Instituto de las Españas, su valioso libro Edgar Allan Poe in Hispanic Literature, que permitió conocer hasta dónde fué estremecida la sensibilidad poética en nuestro idioma, por la del gran poeta cuyo centenario es nuevo motivo para puntualizar esa trascendencia.

Estudiar a Poe sigue siendo preocupación de quienes se interesan por el destino de la Poesía y por una realidad en que concurre la angustia de uno de los espíritus más íntegros de la América que busca en los valores universales una fuente de su propia expresión.

Después del libro de Englekirk han aparecido nuevas interpretaciones sobre Poe y no pocas síntesis para acercarse más a su pensamiento y a su vida. Las of rezco a los estudiosos, con la seguridad de que sabrán aprovecharlas; muy especialmente a quienes siguen en marcha hacia el paraíso prometido de la Poesía.

$$
\text { R. H. V. }
$$

New York-Washington, septiembre, 1949. 


\section{B I B L I O G R A F I A}

\section{TRADUCCIONES}

1922. Eldorado. El Cuervo. Annabel Lee. A Helena. (Versiones de Rafael Lozano.) El Maestro, II (2): pp. 233-240.

1932. Los poemas de Edgard Poe. (Traducción, prólogo y notas por Carlos Obligado.) Buenos Aires, Viau y Zona, 187 pp.

(En la revista Nosotros, de octubre de 1932, xxvi (281): 105-6, hay un comentario bibliográfico de Augusto Cortina, y en él hace mención de anteriores traductores de Poe, al francés y al castellano.)

1937. Edgard Allan Poe. Traducción de sus poemas, crítica y notas (por Alberto L. von Schauenberg). Buenos Aires, Editorial Orientación.

1937. Dos maravillosos relatos de Edgard Allan Poe. Notabilidades y El retrato oval. En Moscú 1937, por Lion Feuchtwanger, Santiago de Chile, Editorial Ercilla.

1941. A Helena. Annabel Lee. Balada nupcial. Las Campanas. El Cuervo. (Traducción de Carlos Obligado.) En Antología de poetas americanos, por Ernesto Morales. Buenos Aires, Santiago Rueda, Editor, pp. 363-373.

1942. Los poemas de Edgard Allan Poe; traducción, prólogo y notas (de Carlos Obligado). Buenos Aires, Espasa-Calpe, Argentina.

(Hay otra edición del mismo año).

1944. Eureka. Marginalia. La filosofía de la composición. (Versión directa de Carlos María Reyles.) Buenos Aires, Emecé editores, 273 pp. (Colección Grandes Ensayistas).

1946. Seven short stories. Edit. by Faye L. Bumpass and Patricia Filliott. Lima, Sanmartí Publishing Co., 193 pp.

Annabel Lee. Revista Moderna de México, 1898, 7: 112.

A Elena. (Traducción de Balbino Dávalos.) En "Los grandes poetas: norteamericanos" Revista Moderna de México, 1901, Iv (21): 336-338; Revista Nueva, Tegucigalpa, $1^{\circ}$ junio, 1902. 
To Helen. (Traducción hecha en prosa.) Jueves del Mundo, México, 19 junio, 1902; y Revista Nueva, Tegucigalpa, $1^{\circ}$ de mayo de 1902 (11)

Annabel Lee. (Traducción de Leopoldo Díaz.) Revista Nueva, Tegucigalpa, $1^{\circ}$ de julio de 1902.

A Helena. (Traducción de Rafael Lozano.) Ateneo de Honduras, 1923 , rv: 1756.

Annabel Lee. (Traducción de Rafael Lozano.) Ateneo de Honduras, 1913, rv: 1755 .

Annabel Lee. (Traducción de Rómulo E. Durón.) Revista del Arcbivo y Biblioteca Nacionales, Tegucigalpa, 1927, vi (7): 251 .

To Helen. (Traducción en prosa por Ernesto Mejía Sánchez), Novedades, 9 octubre, 1949.

(El traductor advierte que dicha versión en prosa apareció por primera vez en español en Revista $A z u l$, México, 4 agosto, 1895; un año después se publicó en El Cojo Ilustrado, de Caracas, y en El Fígaro Mexicano y cuatro años más tarde en Revista Moderna de México. Balbino Dávalos incluyó una traducción en verso, en "Los grandes poetas norteamericanos", México, 1901. Mejía Sánchez cree que es probable que Dávalos sea el autor de la versión en prosa.)

Aurea chinche (de Edgar Allan Poe). (Traducción de Agustín Haro y T.) Los Angeles, Cal., Talleres Linotípicos "La Prensa", 1922.

Dos maravillosos relatos de Edgard Allan Poe. Notabilidades y El retrato oval. Santiago de Chile, Editorial Ercilla, 1937.

El baile. (Ultima creación del celebrado poeta.) Revista Nueva, Tegucigalpa, octubre de 1901.

El Cuervo. (Traducción de José Antonio Pérez Bonalde.) Revista Nacional de Cultura, Caracas, 1946, vir (54): 50-53; "Ariel", San José de Costa Rica, 1942, pp. 3066; y El Diario de Nueva York, octubre 9, 1949.

El Cuervo. (Traducción de Ignacio Mariscal.) El Renacimiento, 1869, I: 158-160; Revista Moderna, México, 1900, III (16): 241-246; El Nacional, México, 1880, I: 45-46; Don Quijote, Puebla, 1910, III (8): 2-3; y Diario de Yucatán, Mérida, 9 octubre de 1949. 
(La traducción la hizo el señor Mariscal en Washington, firmándola el 30 de marzo de 1867.)

El Cuervo. (Traducción de Felipe G. Cazeuneuve.) El Partido Liberal, México, 12 julio, 1885.

El Cuervo. (Versión castellana de Guillermo F. Hall.) El Imparcial, Guatemala, 30 agosto, 1941.

El Cuervo., (Traducido en prosa por R. Gómez Robelo.) Revista Moderna de México, 1904, 7: 26-28.

El Cuervo. Interpretación de Enrique González Martínez. Rueca, México, 1945, rv (15): 11-14.

El escarabajo de oro y otras historias extraordinarias. Buenos Aires, Biblioteca Pluma de Oro, 1939.

El escarabajo de oro. Buenos Aires, Editorial Calomino, 1943.

El escarabajo de oro. Traducción de L. N., Buenos Aires, Editorial Grandes Autores, 1943.

E1 espectro. Don Quijote, Puebla, 1.910, Iv (1): 3-4.

El espectro. El Mundo Ilustrado, México, $1^{\circ}$ enero, 1911.

El gato negro. Traducción de N. L., Buenos Aires, Editorial Grandes Autores, 1943.

El gato negro. Letras del Ecuador. Quito, 1949, v: 3-23.

(No indica el nombre del traductor.)

El retrato oval. El Imparcial, México, 16 mayo, 1898.

El silencio. (Traducción anónima.) Novedades, México, 9 octubre, 1919.

Eldorado. (Traducción de Rómulo E. Durón), Revista del Arcbivo y Biblioteca Nacionales, Tegucigalpa, 1907, m: 610.

Eldorado. (Versión de Rafael Lozano.) Ateneo de Honduras, Tegucigalpa, 1923, tv: 1641.

Enterrado vivo. Revista de Revistas, México, noviembre, 1947. (37) 
Enterrado vivo. ( $\mathrm{L}_{a}$ novela de la semana, en Revista de Revistas.) Mléxico, 1947, 32 pp.

Filosofía de la composición. Revista Moderna de México, noviembre, 1908, pp. 131-139.

Hop-Frog. La Libertad, México, 23 febrero, 1879.

Ifigenia. (La novela de la semana, en Revista de Revistas.) México, $1948,32 \mathrm{pp}$.

La pipa de amontillado. (Traducción por J. A. Pérez Bonalde.) El Domingo, México, 12 enero, 1873.

La barrica de amontillado. Revista Nueva, Tegucigalpa, 15 abril, 1902, I (18): $5-8$.

La canción de J. S. T. Hollands. Cuento inédito. (Traducción de Gustavo A. Baz.) El Domingo, México, 2 junio, 1872, p. 28.

La ciudad en el mar. (Traducción de Leopoldo Díaz.) Revista Nueva, Tegucigalpa, 1902, In (25) : 194; y Ariel, San José Costa Rica, 15 noviembre, 1937.

La durmiente. Jueves de El Mundo, México, 25 septiembre, 1902.

La durmiente. (Traducción de Leopoldo Díaz.) Revista Nueva, Tegucigalpa, 15 agosto, 1902, II (26): 204-205.

La máscara de la "Muerte Roja”. El Nacional, México, 24 julio, 1938.

(Trae al pie una breve nota crítica de Luis Cardoza y Aragón.)

La máscara de la muerte roja. Traducción de Vicente Algarra. Buenos Aires, Editorial Grandes Autores, 1943.

- Las campanas (traducción de Rómulo E. Durón.) Revista de la Universidad, Tegucigalpa, 1914, vi: 6\$2-54.

- Las campanas. (Versión española por el doctor David Cerna.) La Opinión, Los Angeles, California, 1938 (?).

Las campanas. "The bells". Traducción de Jesús Mora Vázquez. vida, Bogotá, mayo, 1941, p. 10. 
Leonina. (Traducción de Rómulo E. Durón.) Revista del Arcbivo y Biblioteca Nacionales, Tegucigalpa, 1905, r: 577-578.

Leonainie. (Traducción de José Juan Tablada.) Revista Moderna de México, febrero, 1906, pp. 368-369.

Leonina. Caras y Caretas, Buenos Aires, 20 febrero, 1937.

Mi pesadilla. Revista Nueva, Tegucigalpa, 1902, II (27): 215. (56)

Sombra. La Libertad, México, 21 noviembre, 1878.

Tierra del sueño. (Traducción de Leopoldo Díaz.) Revista Nueva, Tegucigalpa, 1902, In (29): 227-228.

Tierra del sueño. El Mundo Ilustrado, 14 noviembre, 1909.

(No aparece el nombre del traductor al español. Comienza así: "Por una ruta obscura, por ignorada senda/que recorren los ángeles malditos /).

- Dreamland. (Traducción de Carlos Arturo Torres.) Esfinge, Tegucigalpa, 1917, Xxx (32): 462-463.

Ulalume. (Traducido por Rasch.) Revista Moderna de México, 1898, I (4): $72-73$.

Ulalume. (Traducción de Leopoldo Díaz.) Revista Nueva, Tegucigalpa, 1902, I (22): 170-171.

Ulalume. (Traducido por Alejandro Guanes), Revista Moderna de Mléxico, junio de 1909, pp. 221-223; y El Imparcial, México, 21 junio, 1909.

Un sueño entre un sueño. (Traducción de Carlos Obligado.) La Prensa, Nueva York, 7 octubre, 1949.

\section{SOBRE POE}

Baeza Flores, Alberto. El centenario de Poe, La Prensa, San Antonio Texas, 29 enero y 5 febrero, 1950.

(BAquero, Gastón). El sufrimiento era en Edgar Allan Poe, dijo Gastón Baquero, en la Academia de Letras, camo una transformación, de la que su espíritu de rebelde salía siempre redimido totalmente. Diario de la Marina, 7 mayo, 1949. 
Barba-Jacob, Porfirio. La influencia de Edgar Allan Poe en la poesia colombina de los últimos treinta años. (Apuntamientos para un estudio.) Vida, La Ceiba, (Honduras), 15 marzo, 1918.

Beguin, Albert. Para el centenario de Poe. El Nacional, México, 13 noviembre, 1949; y Letras del Ecuador, Quito, 1949.

Bolaños Cacho, M. Después de la lectura. Edgar Poe. El Renacimiento. (Segunda época.) México, 1894: 122-125; y El Mundo, México, 16 mayo, 1897.

Borges, Jorge Luis. La génesis de "El Cuervo" de Poe. La Prensa, Buenos Aires, 1935. Cervantes, La Habana, 1936, xI: 19-20.

Busto, Jorge del. Edgar Allan Poe. Un grande de la pluma y señor de la fantasía. Crónica, La Habana, $1^{\circ}$ mayo, 1949.

Cardona, Rafael. Y todavía un poco, Edgar Poe. El Nacional, México, 7 mayo, 1938.

Cardoza y Aragón, Luis. Edgar Allan Poe. El Nacional, México, 24 julio, 1938.

Cavazzuttr, Esteban M. Del epistolario de Edgardo Poe y de sus amores. Novedades, Buenos Aires, 1920, xIv (129): 24-44.

Cernta, David. The raven: "El Cuervo" de Edgar Allan Poe y una traducción del licenciado don Ricardo Gómez Robelo. La Prensa, San Antonio de Texas, 9 noviembre, 1942.

Claretie, Jules. Edgar Poe el genio del espanto. Revista de Revistas, 18 septiembre, 1927.

Cómo murió Edgar Allan Poe. La Prensa, Tegucigalpa, 8 enero, 1909.

Cómo murió Edgardo Poe. El Domingo, México, 9 febrero, 1873.

Correa, Luis. Edgar Poe, Pérez Bonalde y José Asunción Silva. Social, Lima, 5 mayo, 1935.

Dávila, Carlos. Poe y el centenario de la novela policial. Diario de la Marina, La Habana, 3 abril, 1947; El Porvenir, Monterrey, 4 abril, 1947; y El Tiempo, Bogotá, 6 abril, 1947. 
Defiende María Clearn la memoria de su yerno, el genial poeta Edgar Poe. Bobemia, La Habana, 1949, xul (49): 133-135.

Dole, N. H. Los últimos amores de Edgar Poe. (Traducción de Arturo Mejía Nieto.) Hispanoamérica, Tegucigalpa, $1,(1) .1^{\circ}$ noviembre, 1922.

Durón Rómulo E. Memoria sobre Edgar Allan Poe. Ateneo de Honduras, 1914, II: 133-134; 169-173.

EchagüE, Juan Pablo. El amor en la literatura. Boletín de la Academia Argentina de Letras, Buenos Aires, 1944, Ix: 473-499.

Edgar Allan Poe y los dirigibles. Una "volada" en dirigible de hace un siglo que resulta verdad en la actualidad. Sucesos, México.

Edgardo Allan Poe. Diario de la Marina, La Habana, 19 octubre, 1941.

En honor de Poe. Diario de la Marina, La Habana, 21 agosto, 1949.

(Es la traducción de un editorial del Times Herald de Washington.)

Englexirk, John Eugene. Edgar Allan Poe in Hispanic Literature. Instituto de las Españas en los Estados Unidos. New York, 1934, 504 pp.

(Un comentario, por Otis H. Green, apareció en Hispanic Review, Philadelphia, 1935, II (2): 173-75.)

Erickson, Martin E. Three Guatemalan translations of Poe. Hispania, Washington, febrero, 1942, pp. 73-78.

EspiNa, Antonio. El celeste Edgardo. Novedades, México, 21 octubre, 1949.

Ferrer, Orlando. Centenario de Poe. La Prensa, New York, 7 octubre, 1949.

Edgard Poe en Nueva York, El Universal Gráfico, México, 30 agosto, 1937.

(Capítulo de La nocbe de Poe.)

Gamboa, Federico. Mi diario. México, 1920, p. 387. 
Godoy, George. Cómo escribió Poe "El Cuervo". El Universal Ilustrado, México, 20 octubre, 1927.

Gómez, Filiberto. Edgar Allan Poe, genio idealista con matices sombríos y diáfanos a la vez. Espíritu rebelde y signo doloroso. El Universal, México, 17 enero, 1948.

Gómez de la Serna, Ramón. El cuervo de Poe. Saber Vivir, Buenos Aires, abril, 1943, pp. 36-37.

GoNZález, Héctor. Notas y apuntes críticos sobre Edgar Allan Poe. El Universal Ilustrado, México, 15 abril, 1920.

González Montesinos, Manuel. La facultad creadora y la estética de Edgar Allan Poe. Novedades, México, 7 octubre, 1949.

González Ramírez, Manuel. Edgar Poe y la perversidad. Noveda$d c s$, México, 9 octubre, 1949.

Greiff, León de. Plegaria a Poe. (Versos.) Diario Latino, San Salvador, 27 agosto, 1938.

Henríquez Ureña, Pedro. Conferencias. El Mundo Ilustrado, 23 octubre, 1910.

(Traza un cuadro de las conferencias en el Ateneo de la Juventud y al referirse a la de Gómez Robelo sobre Poe, dice que señaló "los rasgos esenciales del idealismo trágico de los griegos").

Hernández de Agüero, Fernando. La obra de un "degenerado superior": Edgar Allan Poe. Continente, Lima, octubre noviembre, 1947, pp. 21-22.

Hernández Huidobro, Olga. Edgar Allan Poe y el Cuervo. Noredades, 9 octubre, 1949.

Hispano, Cornelio. La cabaña de Edgar Poe. Social, Lima, 20 marzo, 1935 .

Ichaso, Francisco. Huésped de la niebla. Oyendo a Baquero hablar de Poe. La afición de Poe. Diario de la Marina, 8 mayo, 1949

Ingram, John H. Edgar Allan Poe. Buenos Aires, Ediciones Lautaro, 1944, 347 pp. 
Jaloux, Edmund. Edgar Poe y las mujeres. Buenos Aires, Editorial Argos, 1947.

Kouru, Mario. Edgar Poe. La Opinión, Los Angeles, Cal., 3 febrero, 1950.

L. C. Libros y revistas. Revista Moderna de México, marzo, 1905, pp. 56-57.

(Comenta el libro Edgar Poe, su vida y sus obras por Emilio Lauvrière.)

La fantasía de Edgar Poe. Adivinó el problema de la aviación. El Mundo Ilustrado, México, 5 marzo, 1911.

Laplaza, Francisco P. Edgar Poe, la novela policial y la criminología. Pórtico, Buenos Aires, junio, 1944, pp. 12-14.

Leumann, Carlos Alberto. El raciocinio artístico de Poe. El Nacional, México, 24 agosto, 1941. 1941.

—. El trabajo creador de Poe. La Prensa, Buenos Aires, 8 junio, junio, 1941.

—. Un "canard" filológico de Poe. La Prensa, Buenos Aires, 22

Lillo Catalán, V. Dos grandes sinfonistas opuestos: Rubén Darío y Edgar Poe. La Revista Americana de Buenos Aires, Buenos Aires, 1934, XLIX: $39-43$.

Lozano, Rafael. "Las Campanas" de Edgar Poe. El Universal Ilustrado, México.

Machon, Arthur. El gran dios Pan. Novela de aventuras extraordinarias. Edgard A. Poe. Ligeia. Historia extraordinaria. México, Editorial "Cima", Imprenta de la Escuela "Rafael Dondé", 1940, 222 pp. $1.9 \times 12.5$ cms.

Mallo, Daniel. Poe: el genio trágico de América. El Diario de Nueva York, 9 octubre, 1949.

Marsal, Manuel. El ambiente familiar de Poe. Revista Cubana, La Habana, enero-diciembre, 1944, pp. 166-182. 
Marsicovétere y Durán, M. Las mujeres que amó Edgar Allan Poe. El Imparcial, Guatemala, 18 enero, 1939; y Viento Nuevo, Guatemala, mayo, 1949.

Mathews, Cornelius. Cómo escribió Poe "El Cuervo". El Universal Ilustrado, México, 20 octubre, 1927.

Mejía Sánchez, Ernesto. Edgar Allan Poe en México. Novedades, 9 octubre, 1949.

Mirlares Carlo, Agustín. Historia universal de la Literatura. México, Editorial Esfinge, 1945, p. 408.

Monner Sans, José María. Edgar Allan Poe. La Prensa, Buenos Aires, 2 octubre, 1949.

Montalvo, Antonio. (Comentario sobre) "Los poemas de Edgard Poe" por Carlos Obligado. América, Quito, 1934, IX (54-55): 115-119. bre, 1933.

-

Montesinos Malo, Arturo. La añoranza de lo que nunca fué. Edgar Allan Poe y su tiempo a través de una apasionante reconstrucción biográfica del autor americano Hervey Allen. Letras del Ecuador, Quito, $1949, \vee(50-52): 6-8$.

Müller, Arnaldo. La interesante vida de Edgardo Allan Poe. El Nuevo Diario, Caracas, 10 abril, 1934.

Murphy, Edmund R. Edgard Allan Poe, periodista. Diario Comercral, San Pedro Sula, Honduras, 13 octubre, 1949.

Noulet, Emile. Etudes littéraires. L'hermétisme dans la poesie française moderne. Influence de Edgard Poe sur la poésie française. Mexique, Talleres Gráficos de la Editorial Cultura, 1944, S-13-158 pp.

- Poe en la poesía francesa. Managua, Edit. Nuevos Horizontes, 1944, 25 pp. $11 \times 15 \mathrm{~cm}$. (Cuadernos de literatura extranjera, núm. 6.); Espiral, Bogotá, noviembre, 1949; y El Hijo Pródigo, México, 1943, II (7) : 11-23.

Obligado, Carlos. "El Cuervo". América, Quito (Ecuador), 1934, IX: (54-55): 49-58.

(Es un estudio crítico que acompaña la traducción del poema de Poe.) 
Pedrell, Felipe. Edgardo Poe y la música. El Nacional, México, 5 julio, 1941.

PoE, Edgar Allan. Novedades, México, 9 octubre, 1949.

Reyes Nevárez, Salvador. Edgar Allan Poe y el sentido de lo perverso. Novedades, 9 octubre, 1949.

Rugeles, Manuel F. Canto a Edgar Allan Poe. El Diario de Nueva York, 9 octubre, 1949.

Somerset Maugham, W. El suicidio de Mackintosh. (La novela de la semana, en Revista de Revistas), México, 1948, 31 pp.

Soto Hall, Máximo. Carlos Arturo Torres, traductor de Poe. El Espectador, Bogotá, 13 septiembre, 1926.

- Edgar Allan Poe y sus intérpretes. La Prensa. Buenos Aires.

Tablada, José Juan. Poesía en honor de los poetas norteamericanos. El Imparcial, México, 7 noviembre, 1901.

Torre, Manuel. Presencia de Edgardo Poe. El Nacional, México, 14 agosto, 1941.

Torres-Rroseco, Arturo. El caso de Edgar Allan Poe en la literatura hispanoamericana. Atenea, Santiago, septiembre, 1934, pp. 388-407.

Tosi DE Dréguez, Lola. El principio poético de Edgar Allan Poe. Por nuestro idioma, Buenos Aires, octubre-noviembre, 1941.

Vega, Fausto. La angustia de Poe. Novedades, México, 9 octubre, 1949.

Vela, Arqueles. Edgar Poe. Rueca, México, 1945, iv (15): 15-22.

- Poe: primer brote modernista. En Feoría literaria del modernismo, México, 1949, pp. 25-33.

Vivas, Adolfo. El centenario de Edgar Allan Poe. El Imparcial, México, 20 enero, 1909.

Von HaM, Martha E. Poe: el genio trágico de América. Diario Comercial. San Pedro Sula, 31 diciembre, 1949. 
Woestyn, H. R. Los últimos momentos de Edgar Poe. Nosotros, Buenos Aires, 1924, xvir (187): 503-509.

(Artículo tomado de Les Nouvelles Littéraires de París, 29 noviembre, 1924).

\section{ADDENDA}

\section{TRADUCCIONES}

Annabel Lee, de E. Allan Poe. (Traducción de Guillermo Hall.) En Publicaciones de la Academia Guatemalteca, Guatemala, 1935, m: 49-50.

El cuervo. (Traducción de Jesús Mora Vázquez.) Universidad de Antioquia, Medellín, 1940, $\mathrm{N}^{\circ} 37$.

El pozo y el péndulo. México, (folleto de) Revista de Revistas, $1948,31 \mathrm{pp}$.

El triunfo del gusano. (Traducción de Mariano de Vedia y Mitre.) La Nación, Buenos Aires, 2 octubre, 1949.

(Lleva fecha de septiembre del mismo año, en Buenos Aires).

Las campanas. (Traducción de J. Mora Vázquez.) Revista Javeriana, Bogotá, 1940, xIv: 41-46.

Mi pesadilla. (El último cuento de Poe.) El Mundo Ilustrado, México, 9 marzo, 1902.

Nota sobre "Incidents of travel in Central America, Chiapas and Yucatan", por J. L. Stephens. Grabam Magazine, August, 1841.

Poemas. Con prólogo de Rubén Darío y, su estudio de Baudelaire. Montevideo, Claudio García, 1938, 115 pp.

(Los poemas son traducciones de Alberto Lasplaces, J. A. Pérez Bonalde y Carlos Arturo Torres).

SOBRE POE

Al margen. El Norte, Monterrey, 7 octubre, 1949.

Alcalá, Manuel. Del supuesto materialismo de Poe. Filosofía y Letras, México, 1944, vII (16): 171-184. 
Algarra, Vicente. (Ver No 49.)

Arrieta, Rafael Alberto. Notas sobre el modernismo en Buenos Aires. La Prensa, Buenos Aires, 3 diciembre, 1950.

Balladares, Angela. Edgar Allan Poe. Pandemonium, San José de Costa Rica, 1914, No 119, pp. 674-681.

Baquero, Gastón. El sello de Poe y otras apostillas. Diario de la Marina, $1^{\circ}$ noviembre, 1949.

Baudelaire, Charles. Edgardo (sic) Poe. Su vida y sus obras. Letras del Ecuador, Quito, 1949, v (50-52): 10-13.

Baz, Gustavo A. (Ver No 44.)

Borges, Jorge Luis. Edgar Allan Poe. La Nación, Buenos Aires, 2 octubre, 1949.

Bumpass, Faye L. (Ver N ${ }^{8}$.)

Cardoza y Aragón, Luis. (Ver $\mathrm{N}^{\circ}$ 48.)

Carrión, Alejandro. Danza macabra en la muerte de Poe. Letras del Ecuador, Quito, 1949, v (50-52): 9 y 10.

Cazeuneuve, Felipe G. (Ver $\mathrm{N}^{2}$ 22.)

Cerna, David. (Ver N 51 .)

Cortina, Augusto. (Ver No 2.)

Dávalos, Balbino. (Ver Nos. 10 y 16.)

DíAz, Leopoldo. Edgard Allan Poe (soneto.) El Mundo Ilustrado, México, 27 julio, 1902.

(Ver Nos. 12, 45, 58 y 62).

Duron, Rómulo E. (Ver Nos. 15,35 y 53. ).

Echagüe, Juan Pablo. Edgar Poe y Virginia Clemn. En El amor en la literatura, Buenos Aires, 1941.

Edgardo Poe. El Mundo Ilustrado, México, 29 junio, 1902.

El centenario de Poe. El Mundo Ilustrado, 24 enero, 1909. 
Ferrarr, Santiago A. Edgar Allan Poe, genio narrador. Buenos Aires, Editorial Poseidón, 1947.

FeUchtwanger, Lion. (Ver N ${ }^{\circ} 4$.)

Frgueira, Gastón. Vivencia de Edgar Allan Poe. Voz, México, 1950, I $(15): 63$.

Franco, Luis. Horacio Quiroga ante Poe y Kipling. La Prensa, Buenos Aires, 26 enero, 1947.

Gabriel, José. Edgard Poe poeta contra todos. El Diario de Hoy, (suplemento dominical Centro América), San Salvador, 2 marzo, 1947.

Gómez Robelo, Ricardo. (Ver Nos. 24, 75 y 101.)

González Martínez, Enrique. (Ver N²5.)

GuANEs, Alejandro. (Ver $N^{\circ}$ 63.)

Hall, Guillermo F. (Ver Nos. 23 y 150.)

Haro y T., Agustín. (Ver $\left.\mathrm{N}^{\circ} 17.\right)$

Iduarte, Andrés. En el centenario de Edgar Allan Poe. Repertorio Americano, San José de Costa Rica, $1^{\circ}$ enero, 1950.

Lasplaces, Alberto. (Ver $\left.\mathrm{N}^{9} 157.\right)$

Lazo, Raimundo. Recordación de Poe. Enero, La Habana, I (2): 19-20.

Lozano, Rafael. (Ver Nos. 1, 36, 13 y 14.)

Mariscal, Ignacio. (Ver $\left.\mathrm{N}^{\circ} 21.\right)$

Mejía Nieto, Arturo. (Ver $\mathrm{N}^{9}$ 82.)

Mejía Sánchez, Ernesto. (Ver N 16.)

Méndez de Cuenca, Laura. Annabel Lee. (Versión libre.) El Mundo, México, 14 junio, 1896.

Molina, Juan Ramón. Intimas. (Versos.) El Diario, Tegucigalpa, 6 noviembre, 1897.

("Yo conozco también el cuervo lúgubre / que viera Poe, el soñador maldito"). 
Mora Vázquez, Jesús. (Ver Nos. 52, 151 y 154.)

Obligado, Carlos. (Ver Nos. 2, 5, 6, 64 y 125.)

Pérez Bonalde, José Antonio. (Ver Nos. 20, 42, 79, 157 y 181.)

Precioso hallazgo de Edgardo Poe. Un poema. (Leonina). Diario de Honduras, Tegucigalpa, $1^{\circ}$ enero, 1900.

RASCH. (Ver N $\mathrm{N}^{\circ}$ 61.)

Reyies, Carlos María. (Ver N 7.)

Romero, Héctor Manuel. Las novelas policíacas. Excelsior, 8 abril, 1951.

Salinas, Pedro. Poe in Spain and Spanish America. En Poe in foreign lands and tongues. A symposium. Baltimore, The Johns Hopkins press, 1941.

Sardon, Miguel A. "El Cuervo", de Poe y la versión de Pérez Bonalde. Introducción y notas por... Ipna, Lima, 1945, núm. 4: 66-79.

Schauenberg, Alberto L. von. (Ver N $\mathrm{N}^{3}$.)

Tablada, José Juan. (Ver $\mathrm{N}^{\circ}$ 54.)

Thomson, Augusto (D’Halmar). Edgar Poe. En Los 21, Santiago, 1948.

Torre, Claudio de la. Edgar Allan Poe. Diario de Yucatán, Mérida, 11 diciembre, 1949.

Torres, Carlos Arturo. (Ver Nos. 60, 138 y 157.)

Vanegas, Juan de Dios. La influencia de Poe. La Patria, León (Nicaragua), 1909.

Vedia y Mrtre, Mariano de. (Ver $\mathrm{N}^{\circ} 153$. )

Williams, Stanley T. Edgar Allan Poe: el artista. El Reproductor Campechano, Campeche, 1949, v: 179-201.

Rafael Heliodoro Valle 